\title{
Lack of effect of exercise on the expression profiles of mir-16, mir-21, mir-155, caspase 3 and bcl 2 in a rat model of focal cerebral ischemia
}

\author{
L.B. Porsani ${ }^{1}$, M.L.A. Cirino ${ }^{1}$, F.S. Lizarte Neto $^{1}$, R.Z. Figueiredo ${ }^{1}$, \\ C.A.M. de Carvalho, ${ }^{1,2}$, J.P. da Silva ${ }^{1,3}$, M. T. Durand ${ }^{4}$, P.C. Novais ${ }^{1,5}$, \\ M.F.G.S. Tazima ${ }^{1}$, C.G. Carlotti Jr. ${ }^{1}$, B.O. Colli ${ }^{1}$, D.P.C. Tirapelli ${ }^{1}$ and \\ L.F. Tirapelli ${ }^{1}$ \\ ${ }^{1}$ Departamento de Cirurgia e Anatomia, Universidade de São Paulo, Ribeirão \\ Preto, SP, Brasil \\ ${ }^{2}$ Departamento de Medicina, Centro Universitário Barão de Mauá, Ribeirão \\ Preto, SP, Brasil \\ ${ }^{3}$ Faculdade de Taquaritinga, Taquaritinga, SP, Brasil \\ ${ }^{4}$ Faculdade de Medicina, Universidade de Ribeirão Preto, Medicina, Ribeirão \\ Preto, SP, Brasil \\ ${ }^{5}$ Programa de Pós-graduação em Reabilitação das Interações Estruturais , \\ Universidade de Marília, Marília, SP, Brasil \\ Corresponding author: L.F. Tirapelli \\ E-mail: lab.biomol.cirurgia@fmrp.usp.br
}

Genet. Mol. Res. 19 (4): gmr18594

Received October 24, 2020

Accepted November 12, 2020

Published December 31, 2020

DOI http://dx.doi.org/10.4238/gmr18594

\begin{abstract}
Stroke is a major public health problem, with high mortality rates, and a high frequency of disability. Between 1990 and 2010, stroke increased from the fifth to the third leading cause of disability. In addition, its incidence has increased among younger people, with severe health consequences and increased social costs. There is evidence that physical exercise promotes neuroprotective effects when used as a therapeutic treatment. However, the mechanisms of neuroprotection are not yet well known. The objective of the study was to evaluate the expression profile of microRNAs miR-16, miR-21 and miR-155 and the CASPASE-3 and Bcl-2 genes previously related to apoptosis in the tissue (ischemic focus). Forty-eight Wistar rats were divided into four experimental groups: control group, ischemia group,
\end{abstract}


exercise group and exercise + ischemia group. Before the ischemic procedure, the animals in the exercise and exercise + ischemia groups were submitted to a treadmill training protocol for four weeks. The training lasted $30 \mathrm{~min}$ a day at a speed of $18 \mathrm{~m} / \mathrm{min}$. For real-time PCR analysis, a fragment of the ischemic area was collected from each animal using a punch to analyze the expression of miRNAs; miR16, miR-21, miR-155, CASPASE-3 and Bcl-2 genes. In the animals that had physical exercise, there appeared to be a neuroprotective effect by the action of microRNAs and CASPASE-3, although no significant difference was observed. Further studies are needed to elucidate the role of apoptosis mechanisms in cerebral ischemia associated with physical exercise, as well as the role of microRNAs in the modulation of targets associated with this mechanism.

Key words: Cerebral ischemia; Physical exercise; microRNA; Apoptosis

\section{INTRODUCTION}

Stroke is a major public health problem, with high mortality rates and high levels of disability. Between 1990 and 2010, stroke increased from the fifth to the third leading cause of disability. In addition, its incidence has increased among younger people, with significant health consequences and an increase in social costs (Lappin, 2017). Therapeutic treatments aimed at rehabilitation of ischemic stroke remain limited. In addition, blood perfusion that occurs after ischemia can stimulate oxidative and inflammatory events, causing ischemia / reperfusion injury in the brain (Jouffroy and Vivien, 2017).

There is evidence that physical exercise promotes neuroprotective effects when used as a therapeutic treatment in an experimental ischemia model. However, the mechanisms of neuroprotection are still not well understood (Shamsaei, et al., 2015). Currently, some therapeutic approaches using physical activity are being studied for rehabilitation in experimental cerebral ischemia models. The practice of regular physical exercise can improve quality of life and has been used as a mechanism of neuroprotection. The integration of brain neuronal impulses allows plastic changes that benefit the brain structures that have been compromised due to ischemia injury (Garbuzova-Davis et al., 2013; Cirino et al., 2019).

The intensity of physical exercise also influences neuronal changes. Moderate intensity physical exercise decreases cell apoptosis, increases the neurotrophic factors, and promotes mitochondrial biogenesis and angiogenesis (Chae and Kim, 2009; Chung et al., 2013). When physical exercise precedes cerebral ischemia, it promotes cerebral neuroprotection in rats, and among the mechanisms involved, we can highlight decreased inflammatory reaction, decreased cerebral edema, increased synthesis of brain-derived neurotrophic factor and neuronal growth factor (Damázio et al., 2015). According to Dornbos et al. (2013), the greatest benefit of pre-ischemic exercise is the optimization of the ability to maintain the energy supply.

In an attempt to understand the effects of physical exercise on the body, some biomarkers are being investigated. These include genes associated with various molecular mechanisms, such as differentiation, proliferation and apoptosis, as well as microRNAs that regulate these mechanisms (Hui et al., 2011). 
Jeyaseelan et al. (2008) presented data on the microRNAs expressed in rats submitted to occlusion of the middle cerebral artery. These animals were reperfused for 24 or $48 \mathrm{~h}$ and blood and brain tissue samples were collected. The microRNA expression profile was first described under these conditions, and there is evidence that some microRNAs highly expressed in ischemic brain tissue can be detected in blood samples.

MiR-21 was one of the first human microRNA to be studied and has been extensively investigated. It is involved in various pathophysiological processes related to ischemia and reperfusion, such as the inflammatory process and angiogenesis. Interest in the relationship between miR-21 and ischemia/reperfusion injury has increased in recent years. MicroRNAs have been used as possible biomarkers in studies on apoptosis. Particularly, miR-21 has been shown to be a strong anti-apoptotic factor. It is regulated in several types of cancer, including breast, colorectal and gliomas (Xu et al., 2014).

The expression of miR-15b and miR-16 reduced the level of $\mathrm{Bcl}-2$ protein, suggesting that Bcl-2 is a direct target of these microRNAs. In addition, overexpression of miR-15b or miR-16 can sensitize a gastric cancer cell line to apoptosis (Xia et al., 2008).

MiR-21 plays an important role in reducing cell death caused by cerebral ischemia (Buller et al., 2010). MiR-21 has its effectiveness increased by inhibiting the expression of several genes that have a common apoptotic effect, such as CASPASE 3, and its depletion may cause decreased cell proliferation and increased apoptosis (Saugstad, 2010).

Considerable cerebral infarction and cellular apoptosis is induced by increased expression of miR-155. Inhibitors of miR-155 exhibited protective effects in ischemic stroke, including negative regulation of infarct size in brain tissues in vivo and reduced cell apoptosis (Xing et al., 2016).

The aim of our study was to evaluate the expression levels of microRNAs miR-21, miR-16, miR-155 and the CASPASE-3 and Bcl-2 genes previously associated with apoptosis in rats submitted to a pre-ischemic exercise protocol.

\section{MATERIAL AND METHODS}

The experiments were carried out in accordance with the Ethical Principles for Experimental Animals (COBAO) and the study was approved by the Animal Experimentation Committee (CETEA) of the Ribeirão Preto Medical School - University of São Paulo. Forty-eight adult male Wistar rats (Rattus norvegicus) weighing 280-310g were used. The animals were randomly divided into four experimental groups: control (C): 12 animals sacrificed without undergoing the surgical procedure; ischemic (I): 12 animals submitted to the transient middle cerebral artery occlusion (MCAO) model for $60 \mathrm{~min}$, followed by reperfusion for $24 \mathrm{~h}$ and sacrificed; physical exercise (PE): 12 animals submitted to physical exercise; and ischemic and physical exercise (PE + I): 12 animals submitted to the same treatment as the PE group and focal cerebral ischemia for $60 \mathrm{~min}$, followed by reperfusion for $24 \mathrm{~h}$. Animal weights were recorded weekly.

\section{Training Protocol}

An AVS multiple rats treadmill with a capacity of six rats was used. The speed and inclination of the treadmill was controlled through an electronic panel of the treadmill. The animals in the exercise and exercise + ischemia groups underwent an acclimatization period 
of five days with speeds (5 to $18 \mathrm{~m} / \mathrm{min}$ ) and progressive durations (5 to $15 \mathrm{~min}$ ). The purpose of the adaptation period was to reduce the stress levels associated with the manipulation and use of the treadmill. The protocol consisted of a total period of 4 weeks. The warming up period consisted of $2 \mathrm{~min}$ at a speed of $5 \mathrm{~m} / \mathrm{min}$, gradually increased until reaching the speed of $18 \mathrm{~m} /$ $\mathrm{min}$, in which the animals remained for $30 \mathrm{~min}$ with 0 degree of inclination. For animals that showed signs of fatigue or poor adaptation to the use of the treadmill, the exercise was interrupted. During the training, electric shocks were not applied to the animals, only soft touches with the hands to stimulate them. The animals were removed from the treadmill for recovery when they showed signs of exhaustion. The training was only resumed when the animals were fully recovered.

\section{Induction of Cerebral Ischemia}

The animals were sacrificed at the end of the experimental procedures, $36 \mathrm{~h}$ after the last training session or after the same period for sedentary groups. All animals were partially anesthetized by halothane inhalation and intubated with an orotracheal cannula. Occlusion of the middle cerebral artery (MCAO) was performed through the external carotid artery, which was cranially connected and sectioned for retrograde introduction of a $4 \mathrm{~cm}$ mononylon obstructive wire $2.5 \mathrm{~cm}$ long with an end thickened with silicone by an extension of $5 \mathrm{~mm}$ (Carlotti Jr. et al., 2001). The wire was introduced until it reached the common carotid artery and then progressed cranially through the internal carotid artery until reaching and obstructing the MCA.

\section{RNA extraction and cDNA synthesis}

After removal of the brain, the left cerebral hemisphere cortex was isolated and a sample measuring $7 \mathrm{~mm}$ in diameter was drilled for a biopsy centered along the MCA. The samples were placed in cryovials and stored in liquid nitrogen at $-196^{\circ} \mathrm{C}$ until they were used for RNA extraction.

The total RNA was extracted with the Trizol reagent (Applied Biosystems, USA) according to the manufacturer's instructions. To verify the integrity of the RNA obtained, each sample was subjected to electrophoresis on $1 \%$ agarose gel and subjected to a spectrophotometer that provides the concentration of RNA in a sample of 1 to $2 \mu \mathrm{L}$. In addition to concentration, this device provides values for a ratio related to the integrity of the samples (ratio 260/280). The ideal range to be obtained is 1.7 to 1.9 .

Real-time PCR reactions were performed in duplicate. The amplification was performed in a final volume of $10 \mu \mathrm{L}$, using $5 \mu \mathrm{L}$ of the specific reagent Taqman Master Mix, $0.5 \mu \mathrm{L}$ of each specific probe and $4.5 \mu \mathrm{L}$ of cDNA. A 7500 Real Time PCR System (Applied Biosystems) PCR detection device was used together with the Sequence Detection System software to obtain the $\mathrm{CT}$ values. The data were then exported to Excel spreadsheets to calculate the $\Delta \mathrm{CT}$ values. The GraphPad Prism 4.0 software (GraphPad Prism, Inc, San Diego, CA, USA) was used to generate the graphs and calculate the statistical significance.

U6 was used as an endogenous control for the microRNA and gene reactions, respectively. All reactions were performed in duplicate and analyzed with the 7500 Sequence Detection System (Applied Biosystems).

\section{Statistical Analysis}

Data concerning the microRNAs and genes in the various groups were analyzed statistically by Kruskal-Wallis test followed by the Bonferroni post-test using the GraphPad 
Prism software (GraphPad Software, San Diego, CA, USA). The level of significance was set at $\mathrm{P}<0.05$ for two-tailed tests.

\section{RESULTS}

We did not observe any significant differences in the expression of miRNAs miR-16 ( $\mathrm{P}$ $=0.7513)$, miR-26 $(\mathrm{P}=0.1310), \mathrm{miR}-155(\mathrm{P}=0.0704)$, and Bcl-2 $(\mathrm{P}=0.3600)$ and Caspase-3 $(\mathrm{P}=0.1896)$ in the groups of brain tissues submitted to cerebral ischemia, physical exercise and in the association of the two groups when compared to the control group (Figures 1, 2, 3, 4 and $5)$.

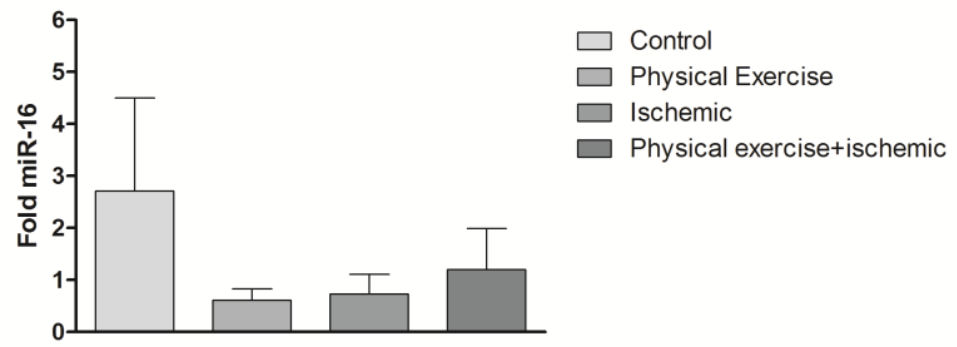

Figure 1. Representation of mean values ( \pm standard error) of microRNA-16 expression in brain tissue of the sedentary and exercise rat groups $(\mathrm{P}=0.7513$, Kruskall Wallis test $)$.

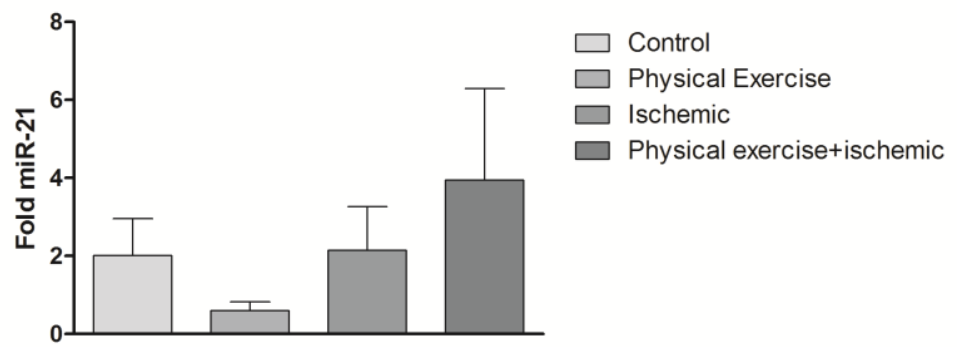

Figure 2. Representation of mean values ( \pm standard error) of the microRNA-21 expression in brain tissue of the sedentary and exercise rat groups $(\mathrm{P}=0.1310$, Kruskall Wallis test $)$.

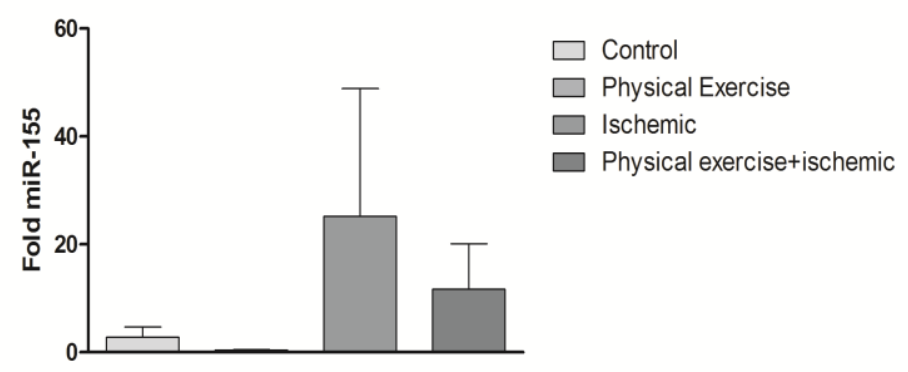

Figure 3. Representation of mean values ( \pm standard error) of the microRNA-155 expression in brain tissue of the sedentary and exercise rat groups $(\mathrm{P}=0.0704$, Kruskall Wallis test $)$. 


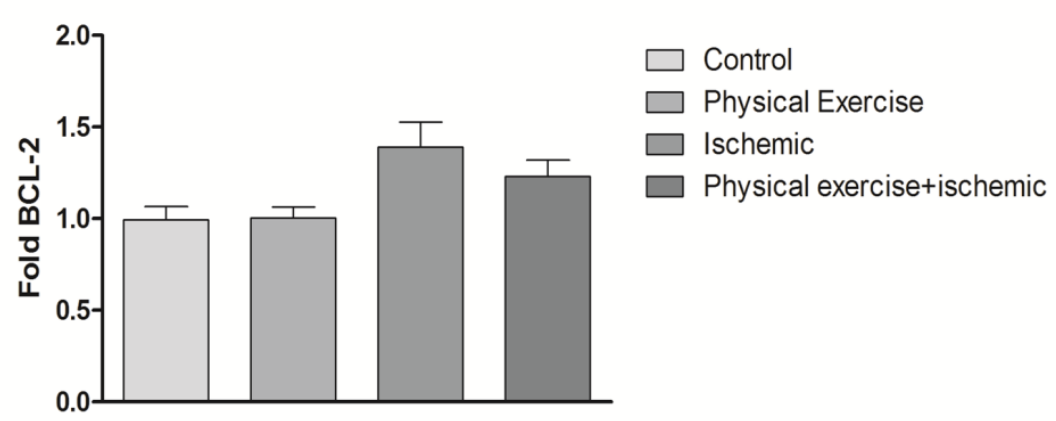

Figure 4. Representation of mean values ( \pm standard error) of the Bcl-2 expression in brain tissue of the sedentary and exercise rat groups $(\mathrm{P}=0.360$, Kruskall Wallis test).

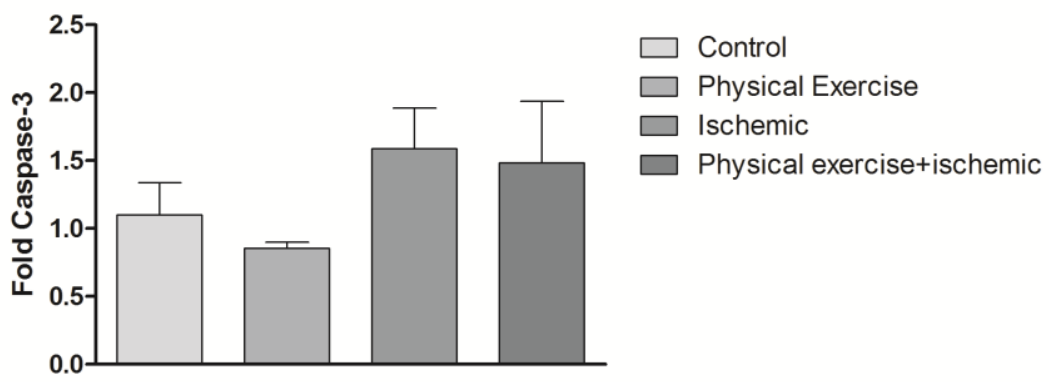

Figure 5. Representation of mean values ( \pm standard error) of the Caspase-3 expression in brain tissue of the sedentary and exercise rat groups $(\mathrm{P}=0.1896$, Kruskall Wallis test $)$.

\section{DISCUSSION}

Evidence shows that regular practice of physical exercise, in addition to promoting the general health of our body, is associated with low risk of mortality and decreased incidence of chronic diseases, such as: cardiovascular diseases, stroke, diabetes mellitus and various types of cancer. In addition, it is known that physical exercise has an influence on the apoptotic process and has been studied as a possible therapeutic method (Warburton et al., 2006).

The aim of our study was to evaluate the expression of some microRNAs and genes that were previously related to apoptosis in research that used animals submitted to the focal cerebral ischemia and exercise model. We expected that animals that were submitted to the exercise protocol on the treadmill before the ischemic procedure, would have a decrease in apoptosis in the ischemic region; however, we did not observe significant differences between the groups.

It is known that the miR-16 family is transcribed from the microRNA-15a/miR16-1 cluster, which inhibits post-ischemic angiogenesis and also function as tumor suppressors by directly associating with BCL-2 (Pekarsky et al., 2018; Besnier et al., 2019). Yang et al. (2017) evaluated the expression profile of microRNAs 15a / 16-1 in a model of cerebral ischemia using adult male mice, performing the administration of antagomirs 15a/ 16-1 intravenously in the animals' tails, submitting these animals to the ischemic procedure 
through MCA occlusion for a period of 1 hour and $72 \mathrm{~h}$ of reperfusion. The results obtained by this study indicated that pharmacological inhibition of microRNAs 15a/16-1 reduces ischemic damage through positive regulation of anti-apoptotic proteins and suppression of pro-inflammatory molecules.

Horak et al. (2018) analyzed the expression of miR-16 in young university students submitted to different training protocols with different intensities during a period of 8 weeks. In this study, the authors concluded that the different intensities studied, such as explosive strength, hypertrophy strength and high-intensity interval training, did not significantly affect expression of miR-16.

Our results support the findings of Horak et al. (2018), since we also did not observe differences in the expression levels of miR-16 in animals submitted to the ischemic procedure associated or not with physical exercise. This suggests that the expression profile of miR-16 is associated with the duration of cerebral ischemia and its reperfusion.

Buller et al. (2010) evaluated the expression of miR-21 in rats after the ischemic procedure and demonstrated that overexpression of miR-21 protects against ischemic neuronal death. Unlike their results, we did not significant effects. This disagreement in expression may be related to the technique used during the ischemic procedure and the duration of ischemia.

Evidence suggests that microRNAs play important roles in the pathogenesis of ischemia and reperfusion injury. Among them, miR-155, as it is a multifunctional microRNA, is highly expressed in epithelial cells and nerve cells. In addition, miRNA-155 is involved in several physiological and pathological processes, such as: differentiation of hematopoietic lineage, immunity, inflammation, cardiovascular diseases, and cancer (Chen et al., 2012).

In the process of ischemic brain injury, miR-155 regulates the expression of proteins associated with various molecular mechanisms, among which we can highlight neuroinflammation and apoptosis (Suofu Y et al., 2020). However, studies demonstrate different findings in the expression profile of miR-155 after ischemic brain injury regulating molecular pathways with opposite effects. In this context, Suofu Y et al., 2020 used knockout mice for the miR-155 and underwent a cerebral ischemia model for 60 min due to occlusion of the middle cerebral artery followed by up to $71 \mathrm{~h}$ of reperfusion. The effects of miR-155 knockout on the size of cerebral infarction, on the incidence and extent of hemorrhage and on neurological evaluation were determined. The results demonstrated an increase in the expression of miR-155 in the cerebral ischemic group in wild-type mice, as well as a reduction in the volume of infarction and neurological deficits in the knockout mice for miR-155. The authors concluded that increased miR-155 expression observed after cerebral ischemia can be considered harmful and its inhibition may be a potential therapeutic target. Our results support the findings of Suofu et al. 2020, since in the ischemic group the expression of miR-155 was elevated and it is still possible to suggest a neuroprotective role for exercise in which it was observed to reduce its expression.

Caspase- 3 is made up of a large family of cysteine proteases that play an essential role in cascade signaling for apoptosis. de Lima et al. (2019) evaluated the effects of preconditioning exercise in an experimental model of renal ischemia and reperfusion in rats. The animals were submitted to the training protocol five days a week, for four weeks, with progressive intensity and duration. Renal IR injury was induced by clamping the bilateral 
renal artery for $45 \mathrm{~min}$. At the end of the study, the animals improved in several aspects and decreased CASPASE-3 levels.

Zhang et al. (2019) using the middle cerebral artery occlusion model, evaluated the neuroprotective effect of exercise (preconditioned for three weeks using Rota-Rod before surgery) on motor performance, infarction volume and the expression of Bax, Bcl- 2 and caspase-3 were measured by Western blot. The results demonstrated the decrease in the volume of the infarction and the expression of Bax and caspase- 3 as well as the increase in the expression of Bcl-2.

Siu et al. (2004) analyzed the levels of BCL-2 and CASPASE-3 in muscle and cardiac tissues in rats submitted to a treadmill running protocol and after $48 \mathrm{~h}$ they found increased levels of Bcl-2 between trained and sedentary groups, but found no significant differences in CASPASE-3 levels. The authors believe that the decreased rate of CASPASE-3 activity may have been influenced by the time taken to collect the material, which was $48 \mathrm{~h}$ after training. Unlike the results reported by de Lima et al. (2019), we found no significant difference, suggesting that the ischemia time or the protocol used in training the animals, may have an influence on gene expression levels.

We did not observe any difference in the expression of CASPASE-3 in the various groups, which allows us to suggest, as also reported by Siu et al. (2004) and de Lima et al. (2019) that the differences in experimental designs both with regard to cerebral ischemia (duration of the ischemic period, time of reperfusion and experimental model of ischemia induction) and physical exercise (model and intensity) may influence the results.

Terashi et al. (2019) evaluated the neuroprotective effects of preconditioning exercise on neuronal apoptosis in rats subjected to focal cerebral ischemia due to occlusion of the left middle cerebral artery lasting $60 \mathrm{~min}$ with reperfusion of $48 \mathrm{~h}$, where several parameters were evaluated: volume from cerebral infarction, neurological function, motor function and Bcl-2, Bax and caspase-3 expression by the TUNEL technique. They found neuroprotective effects of exercise three or more times a week through the regulation of these proteins.

Li et al. (2017) evaluated the role of exercise using Rota-Rod in adult male Sprague-Dawley rats submitted to occlusion of the middle cerebral artery for $2 \mathrm{~h}$. Anti and pro-apoptotic proteins were analyzed using the Western blotting technique. The results showed that the ischemic stroke increased the levels of expression of BAX and caspase-3, and in the ischemic group without associated exercise, apoptotic cell death increased even more, however, only in the group of animals submitted to exercise $6 \mathrm{~h}$ after reperfusion, the same was not observed for reperfusion periods of $24 \mathrm{~h}$ or 3 days. It was also found that Bcl2 expression was not affected by exercise.

Unlike Li et al, (2017), the exercise and exercise + ischemia groups had their relevant microRNA expressions decreased when compared with the sedentary and cerebral ischemia groups.

Physical exercise tended to promote a neuroprotective effect by the action of microRNAs and CASPASE-3 gene, though the differences were not significant. Further studies are needed to elucidate the role of the apoptosis mechanism in cerebral ischemia associated with physical exercise, as well as the role of microRNAs in the modulation of targets associated with this mechanism. 


\title{
ACKNOWLEDGMENTS
}

This study was financed in part by the Coordenação de Aperfeiçoamento de Pessoal de Nível Superior - Brasil (CAPES) - Finance Code 001.

\section{CONFLICTS OF INTEREST}

\author{
The authors declare no conflict of interest.
}

\section{REFERENCES}

Besnier M, Shantikumar S, Anwar M, Dixit P, et al. (2019). Mir-15a/-16 inhibit angiogenesis by targeting the tie2 coding sequence: therapeutic potential of a mir-15a/16 decoy system in limb ischemia. Mol. Ther. Nucleic Acids. 17: 49-62.

Buller B, Liu X, Wang X, Zhang RL, et al. (2010). MicroRNA-21 protects neurons from ischemic death. FEBS J. 277: 4299-4307.

Carlotti Jr. CG, Colli BO and Kazuo JY (2001). Avaliação da isquemia cerebral pela respiração mitocondrial: modelo experimental. Arq. Neuropsiquiatr 59(2-B): 365-371.

Chae CH and Kim HT (2009). Forced, moderate-intensity treadmill exercise suppresses apoptosis by increasing the level of ngf and stimulating phosphatidylinositol 3-kinase signaling in the hippocampus of induced aging rats. Neurochem Int. 55(4): 208-213.

Chung JY, Kim MW, Bang MS and Kim M (2013). Increased expression of neurotrophin 4 following focal cerebral ischemia in adult rat brain with treadmill exercise. Plos One. 8: e52461.

Cirino MLA, Porsani LB, Lizarte Neto FS, Tazima MFGS, et al. (2019). Expressionof miR-15b, miR-29b, miR-219 and miR-222 microRNAs in rats with focal cerebral ischemia submitted to physical exercise. Genet. Mol. Res. 18: gmr18213.

Damázio LCM, Melo RTR, Lima M de C, Pereira VG, et al. (2015). Exercício físico promove neuroproteção estrutural e funcional em ratos com isquemia cerebral. Ver. Neurociênc. 23: 581-588.

de Lima WV, Visona I, Schor N and Almeida W (2019). Preconditioning by aerobic exercise reduces acute ischemic renal injury in rats. Physiol. Rep. e14176.

Dornbos D, Zwagerman N, Guo M, Ding J, et al. (2013). Preischemic exercise reduces brain damage by ameliorating metabolic disorder in ischemia/reperfusion injury. Neurosci. Res. 91: 818-827.

Garbuzova-Davis S, Rodrigues MCO, Hernandez-Ontiveros DG, Tajiri N, et al. (2013). Blood-brain barrier alterations provide evidence of subacute diaschisis in an ischemic stroke rat model. Plos One. 8: e63553.

Horak M, Zlamal F, Lliev R, Kucera J, et al. (2018). Exercise-induced circulating microrna changes in athletes in various training scenarios. Plos One. 13: e0191060. n

Hui A, How C, Ito E and Liu FF (2011). Micro-RNAs as diagnostic or prognostic markers in human epithelial malignancies. BMC Cancer. 11: 500.

Jouffroy R and vivien B (2017). Reply to pang. et al.: "Early detection of brain death using the bispectral index (bis) in patients treated by extracorporeal cardiopulmonary resuscitation (e-cpr) for refractory cardiac arrest". Resuscitation. 12: e9.

Lappin JM, Darke S and Farrell M (2017). Stroke and methamphetamine use in young adults: a review. J. Neurol. Neurosurg Psychiatry. 88: 1079-1091.

Li F, Shi W, Zhao EY, Geng X, et al. (2017). Enhanced apoptosis from early physical exercise rehabilitation following ischemic stroke. J. Neurosci. Res. 95: 1017-1024.

Chen J, Wang B and Tang J (2012). Clinical significance of MicroRNA-155 expression in human breast cancer. $J$. Surgical Oncol. 106: 260-266.

Pekarsky Y, Balatti V and Croce CM (2018). Bcl2 and mir-15/16: from gene discovery to treatment. Cell death differ. 25: $21-26$.

Shamsaei N, Khaksari M, Erfani S, Rajabi H, et al. (2015). Exercise preconditioning exhibits neuroprotective effects on hippocampal ca1 neuronal damage after cerebral ischemia. Neural Rege Res. 10: 1245-1250.

Suofu Y, Wang X, He Y, Li F, et al. (2020). Mir-155 knockout protects against ischemia/reperfusion-induced brain injury and hemorrhagic transformation. Neuroreport. 31: 235-239.

Saugstad AJ (2010). MicroRNAs as effectors of brain function with roles inischemia and injury, neuroprotection, and neurodegeneration. J. Cerebral Blood Flow Metab. 30: 1564-1576.

Siu PM, Bryner RW, Martyn JK and Alway SE (2004). Apoptotic adaptations from exercise training in skeletal and cardiac muscles. FASEB J. 18: 1150-1152. doi:10.1096/fj.03-1291fje. 
Terashi T, Otsuka S, Takada S, Nakanishi K, et al (2019). Neuroprotective effects of different frequency preconditioning exercise on neuronal apoptosis after focal brain ischemia in rats. Neurol. Res. 41:510-518.

Jeyaseelan K, Lim KY and Armugam A (2008). MicroRNA Expression in the Blood and Brain of Rats Subjected to Transient Focal Ischemia by Middle Cerebral Artery Occlusion. Stroke. 39: 959-966.

Xing G, Luo Z, Zhong C, Pan X. et al. (2016). Influence of miR-155 on Cell Apoptosis in Rats with Ischemic Stroke: Role of the Ras Homolog Enriched in Brain (Rheb)/mTOR Pathway. Med. Sci. Monit. 22: 5141-5153.

Xu X, Kriegel AJ, Jiao X, Liu H. et al. (2014). Mir-21 in ischemia/reperfusion injury: a double-edged sword? Physiol. Genomics. 46: 789-97.

Yang X, Tang X, Sun P, Shi Y, et al. (2017). Microrna-15a/16-1 antagomir ameliorates ischemic brain injury in experimental stroke. Stroke. 48: 1941-1947.

Xia L, Zhang D, Du R, Pan Y, et al. (2008). miR-15b and miR-16 modulate multidrug resistance by targeting BCL2 in human gastric cancer cells. Int. J. Cancer. 123: 372-379.

Zhang Z, Li R, Zhang X, Wei Y, et al. (2019). Voluntary exercise promotes neurotrophic factor and suppresses apoptosis in hippocampal ischemia. Jf. Integr. Neurosci. 18: 65-70.

Warburton DE, Nicol CW and Bredin SS (2006). Health benefits of physical activity: the evidence. CMAJ. 174: 801-9.

Genetics and Molecular Research 19 (4): gmr18594 